\title{
Correlation between Chemokine Receptor CXCR4 Expression and Prognostic Factors in Patients with Prostate Cancer
}

\author{
Seok Jin Jung, Chun Il Kim, Choal Hee Park, Hyuk Soo Chang, Byung Hoon Kim, Mi Sun Choi, \\ Hyea Ra Jung
}

Department of Urology, Dongsan Medical Center, Keimyung University School of Medicine, Daegu, Korea

\begin{abstract}
Purpose: We evaluated the correlation between the expression of CXCR4 and prognostic factors in patients with prostate cancer.

Materials and Methods: A total of 57 patients who had undergone surgery for prostate cancer were enrolled. Specimens were obtained before any treatment and were stained with antihuman CXCR4 antibody. The intensity of staining was graded as low or high. The age, pretreatment prostate-specific antigen (PSA) level, Gleason score, T stage, biochemical recurrence, local recurrence, and distant metastasis were compared according to the expression of CXCR4 in patients with prostate cancer.

Results: Local recurrence was higher in the group with high expression, in 11 of 36 cases (30.6\%), than in the group with low expression, in 1 of 21 cases (4.8\%), with statistical significance $(\mathrm{p}=0.040)$. Distant metastasis was also associated with expression, occurring in 10 of 36 cases (27.8\%) in the group with high expression and in 1 of 21 cases (4.8\%) in the group with low expression ( $\mathrm{p}=0.041$ ). In the logistic regression test, CXCR4 expression was the only factor in determining local recurrence $(\mathrm{p}=0.016)$ and distant metastasis (0.022). Furthermore, the group with high CXCR4 expression showed significantly longer cancer-specific survival than did the low expression group $(p=0.041)$. CXCR4 showed no association with age $(\mathrm{p}=0.881)$, pretreatment PSA level $(\mathrm{p}=0.584)$, Gleason score $(\mathrm{p}=0.640), \mathrm{T}$ stage $(\mathrm{p}=0.967)$, or biochemical recurrence $(\mathrm{p}=0.081)$.

Conclusions: The high expression of CXCR4 was associated with local recurrence and distant metastasis. CXCR4 expression was shown to be a useful prognostic factor for patients with prostate cancer.
\end{abstract}

Key Words: CXCR4; Receptor, Prognosis; Prostate neoplasms

This is an Open Access article distributed under the terms of the Creative Commons Attribution Non-Commercial License (http://creativecommons.org/licenses/by-nc/3.0) which permits unrestricted non-commercial use, distribution, and reproduction in any medium, provided the original work is properly cited.

\author{
Article History: \\ received 10 June, 2011 \\ accepted 4 August, 2011
}

\section{INTRODUCTION}

Prostate cancer is the most commonly diagnosed visceral cancer in men and is responsible for the second highest cancer-related male mortality rate in Western countries, with increasing rates being reported in Korea, Japan, and China [1]. Prostate cancer-related deaths are not the result of primary tumor growth but rather are caused by the spread of the cancer to other organs. Cancer metastasis is a sequential, highly selective process induced by growth factors, cell adhesion molecules, and chemoattractants [2]. It has been shown that CXCL12-CXCR4 interactions may play a sig- nificant role in cancer metastasis, and CXCL12 and CXCR4 have been implicated in the pathogenesis and progression of various cancers [3-5].

CXCR4 is one of the chemokine receptors that are being actively studied. It plays the role of a co-receptor of HIV entry and also a role in controlling metastasis in various tumors such as prostatic cancer. A large amount of CXCL12 is formed in organs where tumor metastasis frequently occurs, such as liver, lung, and bones [6]. The tumor cells that manifest CXCR4, which is a receptor of the same family as CXCL12, move to these organs to combine with CXCL12, causing cancer metastasis. 
Studies of the correlation of the CXCL12-CXCR4 ligand-receptor system with the pathology and prognosis of various tumors are currently being conducted. For example, correlations of the manifestation of CXCR4 with lung cancer, melanoma, esophageal cancer, and ovarian cancer have been reported [7-10]. Furthermore, it was reported that the manifestation of CXCR4 in prostatic cancer was associated with low cancer-specific survival [11], and that the CXCL12-CXCR4 interaction played an important role in the bone metastasis of prostatic cancer [3].

To investigate the effect of the manifestation of CXCR4 on prostatic cancer, we examined the relationship between CXCR4 and the prognostic factors of prostatic cancer.

\section{MATERIALS AND METHODS}

\section{Subjects}

The subjects of this study were 57 patients with a diagnosis of prostate cancer who underwent retropubic radical prostatectomy between January 2001 and February 2008. The age of the patients, pretreatment prostate-specific antigen (PSA) level, Gleason score, T stage, biochemical recurrence, local recurrence, and distant metastasis during the follow-up period were examined, and the average follow-up period was 39 months (range, 12 to112 months). One pathologist evaluated the stage of the primary tumor and the Gleason score of the tumors by observing samples stained with H\&E under an optical microscope. Biochemical recurrence was defined as an increase in PSA above 0.2 $\mathrm{ng} / \mathrm{ml}$, twice or more in succession, during the follow-up period.

\section{Immunohistochemical staining}

A pathologist reexamined the H\&E stained sample of the target case and marked the representative tumor site. A core with a $3 \mathrm{~mm}$ diameter was collected from the corresponding paraffin block and a tissue microarray (TMA) was produced. The TMA paraffin block was cut into a slice with a thickness of $4 \mu \mathrm{m}$ and attached to a slide. It was deparaffinized for 5 minutes in Xylene three times and passed through an anhydrous process before being washed with distilled water. The TE buffer solution ( $\mathrm{pH}$ 9.0) was used as a pretreatment for restoration of antigenicity. The sample was stained with the Autostainer 360 (Lab Vision, Fremont, CA, USA) using UltraVision LP Detection (Thermo Scientific, Waltham, MA, USA). For the primary antibody response, CXCR4 (sc-6191, Santa Cruz Biotechnology, Santa Cruz, CA, USA) was diluted at 1:100 and reacted for 40 minutes at room temperature. For contrast stain, Mayer's hematoxylin was used.

\section{Evaluation of immunohistochemical staining}

Two pathologists each interpreted the staining without prior knowledge of any of the clinicopathologic parameters. If the pathologists' analysis did not correspond, the staining was interpreted again. A judgement was made as to the proper immunohistochemical score of the tumors on the basis of the intensity of CXCR4 expression as follows: negative, weak, moderate, and strong staining. Samples with immunohistochemical scores of negative or weak were considered to have low expression and those with moderate to strong distributions were considered to have high expression of CXCR4 antibodies (Fig. 1).

\section{Statistical analysis}

The Gleason score ( 6 or lower, 7 or higher), pretreatment PSA level, T stage, biochemical recurrence, local recurrence, distant metastasis during the follow-up period,
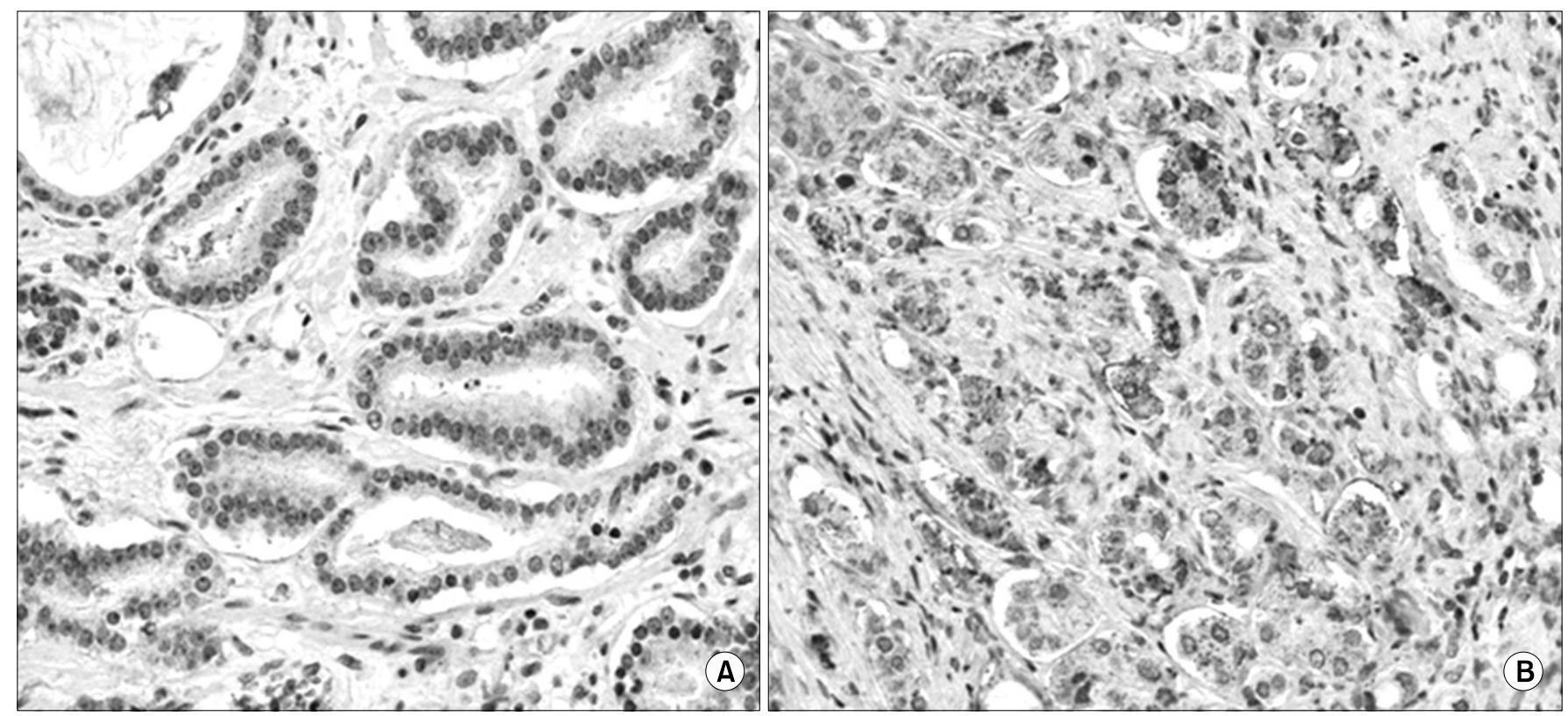

FIG. 1. CXCR4 expression in prostate cancer tissue (anti-CXCR4 antibody, x200). (A) Low expression, (B) High expression. 
and cancer-specific survival were compared according to the expression of CXCR4 in prostate cancer. Statistical significance was analyzed by using the Student's t-test, chi-square test, and logistic regression analysis with SPSS ver. 12.0 (SPSS Inc., Chicago, IL, USA). Cancer-specific survival was calculated by the Kaplan-Meier method. Statistical significance was based on a p-value less than 0.05 .

\section{RESULTS}

\section{Clinicopathologic characteristics}

The mean age of the patients was 64 years (range, 51 to 76 years) and their mean pretreatment PSA level was 7.6 $\mathrm{mg} / \mathrm{ml}$ (range, 3.2 to $18.4 \mathrm{ng} / \mathrm{ml}$ ) (Table 1). Twenty-four patients had a Gleason score of 6 or lower $(42.1 \%)$ and 33 cases had a score of 7 or higher (57.9\%). T stages were as follows: $\mathrm{T} 2$ in 49 cases $(86.0 \%)$, T3 in 8 cases $(14.0 \%)$, and $\mathrm{T} 4$ in 0 cases. Biochemical recurrence was detected in 19 cases $(33.3 \%)$, local recurrence in 12 cases $(21.1 \%)$, and distant metastasis in 11 cases $(19.3 \%)$. Positive surgical margins were detected 8 cases ( $14.0 \%$; low; 3 ; high, 5 ), and there was no significant difference between the 2 groups $(\mathrm{p}=0.215)$.

\section{Expression profiles of CXCR4}

CXCR4 was detected in $93.0 \%$ of the patients (53/57) with prostate cancer specimens (Table 2). Among all patients, the group with low CXCR4 expression contained 21 patients (36.8\%; negative, 4 ; weak, 17$)$, and the group with high expression contained 36 patients (63.2\%; moderate, 30; strong, 6).

Local recurrence of prostate cancer was higher in the group with high expression $(30.6 \%, 11 / 36)$ than in the group

TABLE 1. Clinicopathological characteristics of the patients

\begin{tabular}{lc}
\hline \multicolumn{1}{c}{ Variables } & \\
\hline Mean age $(\mathrm{yr})$ & $64(51-76)$ \\
Mean pretreatment PSA $(\mathrm{ng} / \mathrm{ml})$ & $7.6(3.2-18.4)$ \\
Gleason score & No. of patients $(\%)$ \\
$\leq 6$ & $24(42.1)$ \\
$\geq 7$ & $33(57.9)$ \\
T stage & $49(86.0)$ \\
T2 & $8(14.0)$ \\
T3 & $0(0)$ \\
T4 & \\
Biochemical recurrence & $38(66.7)$ \\
No & $19(33.3)$ \\
Yes & \\
Local recurrence & $45(78.9)$ \\
No & $12(21.1)$ \\
Yes & \\
Distant metastasis & $46(80.7)$ \\
No & $11(19.3)$ \\
Yes & $8(14.0)$ \\
Positive surgical margin &
\end{tabular}

PSA: prostate-specific antigen with low expression $(4.8 \%, 1 / 21 ; \mathrm{p}=0.040)$. Distant metastasis was also associated with expression: it occurred in $27.8 \%$ (10/36) of the group with high expression and $4.8 \%$ $(1 / 21)$ of the group with low expression $(\mathrm{p}=0.041)$. In the logistic regression analysis, CXCR4 expression was the only factor affecting local recurrence $(\mathrm{p}=0.016)$ and distant metastasis $(\mathrm{p}=0.022)$ among age, pretreatment PSA, Gleason score, and CXCR4. Furthermore, the high CXCR4 expression group showed significantly longer cancer-specific survival than did the low expression group $(\mathrm{p}=0.041)$ (Fig. 2).

CXCR4 showed no association with age $(\mathrm{p}=0.881)$, pretreatment PSA level $(\mathrm{p}=0.584)$, Gleason score $(\mathrm{p}=0.640), \mathrm{T}$ stage $(p=0.967)$, or biochemical recurrence $(p=0.081)$.

\section{DISCUSSION}

The soluble attractant molecule, chemokine, was found to play a role in metastasizing specific tumors to other organs [6]. This chemokine is composed of small, basic chemotactic proteins that play a role in its function by combining with G-protein-coupled receptor (chemokine receptor) [12]. This was found to play a role in the direct extravasation in inflammatory cells [13]. Just as leukocytes gather to inflammatory cells, tumor cells move because these chemokines want to combine with chemokine receptors [14-16].

There have been many studies on the role of the CXCL12-CXCR4 ligand-receptor system in immunology and infection areas such as hematopoiesis, lymphocyte homing, and HIV infection. In 2001, Müller et al demon-

TABLE 2. Comparison of clinicopathological characteristics according to CXCR4 expression

\begin{tabular}{lccc}
\hline & \multicolumn{2}{c}{ CXCR4 expression } & \\
\cline { 2 - 3 } Variables & Low & High & p-value \\
& $(\mathrm{n}=21)$ & $(\mathrm{n}=36)$ & \\
\hline Age (yr) & $65.1 \pm 11.5$ & $63.5 \pm 10.7$ & $0.881^{\mathrm{a}}$ \\
Mean pretreatment PSA & $7.2 \pm 4.2$ & $8.5 \pm 3.9$ & $0.584^{\mathrm{a}}$ \\
(ng/ml) & & & \\
Gleason score & & & \\
$\leq 6(\%)$ & $13(38.1)$ & $16(44.4)$ & $0.640^{\mathrm{b}}$ \\
$\geq 7(\%)$ & $20(55.6)$ & \\
T stage & $18(85.7)$ & $31(86.1)$ & $0.967^{\mathrm{c}}$ \\
T2 (\%) & $3(14.3)$ & $5(13.9)$ & \\
T3 (\%) & & & \\
Biochemical recurrence & $17(81.0)$ & $21(58.3)$ & $0.081^{\mathrm{b}}$ \\
No (\%) & $4(19.0)$ & $15(41.7)$ & \\
Yes (\%) & & & \\
Local recurrence & $20(95.2)$ & $25(69.4)$ & $0.040^{\mathrm{c}}$ \\
No (\%) & $1(4.8)$ & $11(30.6)$ & \\
Yes (\%) & & & \\
Distant metastasis & $20(95.2)$ & $26(72.2)$ & $0.041^{\mathrm{c}}$ \\
No (\%) & $1(4.8)$ & $10(27.8)$ & \\
Yes (\%) & &
\end{tabular}

PSA: prostate-specific antigen, ${ }^{a}$ : student's t-test, ${ }^{\text {b}}$ : Pearson chi-square test, ${ }^{\mathrm{c}}:$ Fisher's exact test 


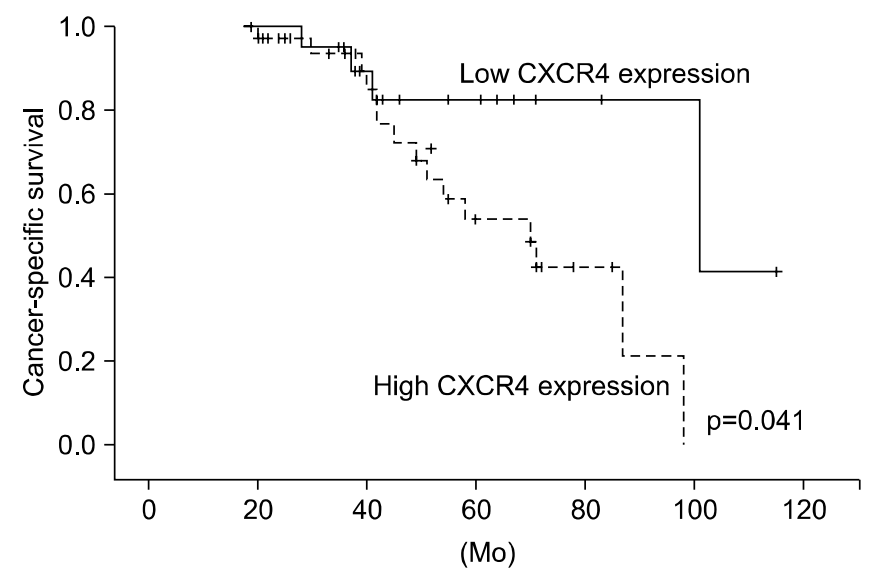

FIG. 2. Kaplan-Meier univariate survival analysis in patients with prostate cancer according to CXCR4 expression.

strated that CXCR4 manifested much more in breast cancer tissues than in normal breast tissues [6]. They also showed that CXCL12 manifested in organs in which breast cancer metastasis frequently occurs, such as lymph nodes, bone marrow, and lung, but did not manifest in kidney, where metastasis seldom occurs. Moreover, an in vivo study found that injecting antibodies neutralizing the CXCR4 activity decreased metastasis [6]. These reports suggest that the CXCL12-CXCR4 ligand-receptor system plays an important role in determining the metastatic destination of cancer cells. Similar results were reported for tumors such as esophageal tumors, malignant melanoma, ovarian cancer, and lung cancer [9,17-19]. However, such studies have not yet been actively conducted for prostate cancer.

In a previous study on prostate cancer and CXCR4, Mochizuki et al reported that positive CXCR4 protein was found in $57.1 \%$ of prostatic samples and stated that the CXCR4 manifestation is an independent predictor of bone metastasis and was better for prediction of bone metastasis than was the Gleason score [20]. A study on metastatic prostate cancer found that the high CXCR4 manifestation group showed poorer cancer-specific survival compared with the low CXCR4 manifestation group [11]. Our study investigated the correlations of CXCR4 manifestation with clinicopathological factors, recurrence, and metastasis and found that CXCR4 manifestation was related to local recurrence and distant metastasis. We confirmed that CXCR4 was the only factor affecting local recurrence and distant metastasis, and the high CXCR4 expression group showed longer cancer-specific survival than did the low expression group. These results support the fact that CXCR4 is a useful prognostic factor in prostate cancer, and the CXCL12-CXCR4 ligand-receptor system plays an important role in the metastasis of prostate cancer to other organs. In all studies, however, CXCR4 had no correlation with the important predictors of the recurrence of prostatic cancer, such as pretreatment PSA level, clinical stage, and Gleason score. The reason for this may be the existence of detection bias because immunohistochemical staining was performed for only a part of prostate cancer specimens. More studies with a larger number of cases are needed to confirm the significance of CXCR4 in prostate cancer.

The fact that CXCR4 is a significant prognostic factor in prostate cancer patients and that the manifestation of CXCR4 indicates poor prognosis suggest that inhibiting CXCR4 could be a possible therapeutic target. In fact, in vitro, anti-CXCR4 antibodies used in therapeutic strategies in a prostate cancer cell line are largely classified into two categories: antibody for CXCR4 and specific CXCR4 antagonist. It was first reported that in the severe combined immunodeficiency (SCID) mouse model, the injection of the antihuman CXCR4 monoclonal antibody restricted the lung metastasis of human breast cancer [6]. This antibody was also found to restrict lung metastasis in murine B16 melanoma and bone metastasis of human prostate cancer in the murine model $[19,21]$. Recently, however, the CXCR4 antagonist was suggested to be a promising therapy for cancer metastasis. AMD3100 is the most powerful and selective CXCR4 antagonist that has been discovered to date [22]. AMD3100 effectively restricted the growth of brain tumor (glioblastoma cell) that had been injected into mice and also increased the apoptosis of cells [23]. T140, another CXCR4 antagonist, was found to decrease pulmonary metastasis of human breast cancer cells in SCID mice [24]. Both AMD3100- and T140-derived CXCR4 antagonists were found to be active in the animal tumor model, providing the basis for later clinical experiments using this drug in many tumor patients $[25,26]$.

Efforts at understanding the role of CXCR4 in cancer biology and finding treatment methods by inhibiting the CXCR4 mechanism may provide novel treatment approaches.

\section{CONCLUSIONS}

The high expression of CXCR4 was associated with local recurrence and distant metastasis. According to our study, CXCR4 expression is a useful prognostic factor for patients with prostate cancer. More studies with a larger number of cases are needed to confirm the clinical significance of CXCR4 in prostate cancer.

\section{Conflicts of Interest}

The authors have nothing to disclose.

\section{REFERENCES}

1. Roberts MJ, Schirra HJ, Lavin MF, Gardiner RA. Metabolomics: a novel approach to early and noninvasive prostate cancer detection. Korean J Urol 2011;52:79-89.

2. Wang J, Xi L, Hunt JL, Gooding W, Whiteside TL, Chen Z, et al. Expression pattern of chemokine receptor 6 (CCR6) and CCR7 in squamous cell carcinoma of the head and neck identifies a novel metastatic phenotype. Cancer Res 2004;64:1861-6.

3. Taichman RS, Cooper C, Keller ET, Pienta KJ, Taichman NS, 
McCauley LK. Use of the stromal cell-derived Factor-1/CXCR4 pathway in prostate cancer metastasis to bone. Cancer Res 2002;62:1832-37.

4. König JE, Senge T, Allhoff EP, König W. Analysis of the inflammatory network in benign prostate hyperplasia and prostate cancer. Prostate 2004;58:121-9.

5. Engl T, Relja B, Blumenberg C, Müller I, Ringel EM, Beecken WD, et al. Prostate tumor CXC-chemokine profile correlates with cell adhesion to endothelium and extracellular matrix. Life sci 2006;78:1784-93.

6. Müller A, Homey B, Soto H, Ge N, Catron D, Buchanan ME, et al. Involvement of chemokine receptors in breast cancer metastasis. Nature 2001;410:50-6.

7. Spano JP, Andre F, Morat L, Sabatier L, Besse B, Combadiere C, et al. Chemokine receptor CXCR4 and early-stage non-small cell lung cancer: pattern of expression and correlation with outcome. Ann Oncol 2004;15:613-7.

8. Scala S, Ottaiano A, Ascierto PA, Cavalli M, Simeone E, Giuliano $\mathrm{P}$, et al. Expression of CXCR4 predicts poor prognosis in patients with malignant melanoma. Clin Cancer Res 2005;11:1835-41.

9. Koishi K, Yoshikawa R, Tsujimura T, Hashimoto-Tamaoki T, Kojima S, Yanagi H, et al. Persistent CXCR4 expression after preoperative chemoradiotherapy predicts early recurrence and poor prognosis in esophageal cancer. World J Gastroenterol 2006;12: 7585-90.

10. Jiang YP, Wu XH, Shi B, Wu WX, Yin GR. Expression of chemokine CXCL12 and its receptor CXCR4 in human epithelial ovarian cancer: an independent prognostic factor for tumor progression. Gynecol Oncol 2006;103:226-33.

11. Akashi T, Koizumi K, Tsuneyama K, Saiki I, Takano Y, Fuse H. Chemokine receptor CXCR4 expression and prognosis in patients with metastatic prostate cancer. Cancer Sci 2008;99:539-42.

12. Zlotnik A, Yoshie O. Chemokines: a new classification system and their role in immunity. Immunity 2000;12:121-7.

13. Foster SJ, Aked DM, Schröder JM, Christophers E. Acute inflammatory effects of a monocyte-derived neutrophil-activating peptide in rabbit skin. Immunology 1989;67:181-3.

14. Constantin G, Majeed M, Giagulli C, Piccio L, Kim JY, Butcher EC, et al. Chemokines trigger immediate beta2 integrin affinity and mobility changes: differential regulation and roles in lymphocyte arrest under flow. Immunity 2000;13:759-69.

15. Mitchison TJ, Cramer LP. Actin-based cell motility and cell locomotion. Cell 1996;84:371-9.

16. Andrew DP, Spellberg JP, Takimoto H, Schmits R, Mak TW, Zukowski MM. Transendothelial migration and trafficking of leukocytes in LFA-1-deficient mice. Eur J Immunol 1998;28:195969.

17. Payne AS, Cornelius LA. The role of chemokines in melanoma tumor growth and metastasis. J Invest Dermatol 2002;118:915-22.

18. Scotton CJ, Wilson JL, Scott K, Stamp G, Wilbanks GD, Fricker S, et al. Multiple actions of the chemokine CXCL12 on epithelial tumor cells in human ovarian cancer. Cancer Res 2002;62:5930-8.

19. Kijima T, Maulik G, Ma PC, Tibaldi EV, Turner RE, Rollins B, et al. Regulation of cellular proliferation, cytoskeletal function, and signal transduction through CXCR4 and c-Kit in small cell lung cancer cells. Cancer Res 2002;62:6304-11.

20. Mochizuki H, Matsubara A, Teishima J, Mutaquchi K, Yasumoto $\mathrm{H}$, Dahiya R et al. Interaction of ligand-receptor system between stromal-cell-derived factor- 1 and CXC chemokine receptor 4 in human prostate cancer: a possible predictor of metastasis. Biochem Biophys Res Commun 2004;30:656-63.

21. Murakami T, Maki W, Cardones AR, Fanq H, Tun Kyi A, Nestle FO, et al. Expression of CXC chemokine receptor-4 enhances the pulmonary metastatic potential of murine B16 melanoma cells. Cancer Res 2002;62:7328-34.

22. De Clercq E. Potential clinical applications of the CXCR4 antagonist Bicyclam AMD3100. Mini Rev Med Chem 2005;5:805-24.

23. Rubin JB, Kung AL, Klein RS, Chan JA, Sun Y, Schmidt K, et al. A small-molecule antagonist of CXCR4 inhibits intracranial growth of primary brain tumors. Proc Natl Acad Sci U S A 2003;100:13513-8.

24. Tamamura H, Hori A, Kanzaki N, Hiramatsu K, Mizumoto M, Nakashima H, et al. T140 analogs as CXCR4 antagonists identified as anti-metastatic agents in the treatment of breast cancer. FEBS Lett 2003;28:79-83.

25. Smith MC, Luker KE, Garbow JR, Prior JL, Jackson E, Piwnica-Worms D, et al. CXCR4 regulates growth of both primary and metastatic breast cancer. Cancer Res 2004;64:8604-12.

26. Takenaga M, Tamamura H, Hiramatsu K, Nakamura N, Yamaguchi Y, Kitagawa A, et al. A single treatment with microcapsules containing a CXCR4 antagonist suppresses pulmonary metastasis of murine melanoma. Biochem Biophys Res Commun 2004;320:226-32. 